\title{
Presynaptic Protein Kinase Activity Supports Long-Term Potentiation at Synapses Between Individual Hippocampal Neurons
}

\author{
Paul Pavlidis, Johanna Montgomery, and Daniel V. Madison \\ Department of Molecular and Cellular Physiology, Stanford University School of Medicine, Stanford, \\ California 94305-5345
}

Simultaneous microelectrode recording from two individual synaptically connected neurons enables the direct analysis of synaptic transmission and plasticity at a minimal synaptic connection. We have recorded from pairs of CA3 pyramidal neurons in organotypic hippocampal slices to examine the properties of long-term potentiation (LTP) at such minimal connections. LTP in minimal connections was found to be identical to the NMDA-dependent LTP expressed by CA3-CA1 synapses, demonstrating this system provides a good model for the study of the mechanisms of LTP expression. The LTP at minimal synaptic connections does not behave as a simple increase in transmitter release probability, because the amplitude of unitary EPSCs can increase several-fold, unlike what is observed when release probability is increased by raising extracellular calcium. Taking advantage of the relatively short axon connecting neighboring CA3 neurons, we found it feasible to introduce pharmacological agents to the interior of presynaptic terminals by injection into the presynaptic soma and have used this technique to investigate presynaptic effects on basal transmission and LTP. Presynaptic injection of nicotinamide reduced basal transmission, but LTP in these pairs was essentially normal. In contrast, presynaptic injection of $\mathrm{H}-7$ significantly depressed LTP but not basal transmission, indicating a specific role of presynaptic protein kinases in LTP. These results demonstrate that pharmacological agents can be directly introduced into the presynaptic cell and that a purely presynaptic perturbation can alter this plasticity.

Key words: long-term potentiation; presynaptic; protein kinase; hippocampus; electrophysiology; synaptic transmission
Long-term potentiation (LTP) of synaptic transmission has been widely studied as a potential substrate of learning and memory at the synaptic level of neuronal circuitry (Bliss and Collingridge, 1993). Despite considerable effort, the understanding of the cellular and molecular underpinnings of this form of synaptic plasticity is still limited. In particular, the specific roles of the presynaptic and postsynaptic elements of the synapse have been difficult to separate and characterize. In general, it has been easier to study the role of the postsynaptic side of the synapse because of the ability to place microelectrodes into the postsynaptic cell and to introduce pharmacological agents exclusively there (Lynch et al., 1983; Malinow et al., 1989; Lledo et al., 1995). Measurements of presynaptic function in LTP have been primarily indirect because of the inaccessibility of small presynaptic terminals, although new techniques are beginning to be brought to bear on this side of the synapse (cf. Ryan et al., 1997).

We have sought to directly assay a role of the presynaptic terminal in the induction, maintenance, or expression of LTP by recapitulating the critical feature of experiments that have been used so successfully to assay postsynaptic function in these phases

Received Feb. 1, 2000; revised April 6, 2000; accepted April 7, 2000.

This work was supported by the Silvio Conte-National Institute of Mental Health Center for Neuroscience Research Grant MH48108. We would like to thank Dominique Muller for his generous assistance with the interface organotypic slice preparation. We would also like to thank Eric Schaible for his excellent technical assistance on this project.

Correspondence should be addressed to Dr. Daniel V. Madison, Department of Molecular and Cellular Physiology, Beckman Center, Room 111b, Stanford University School of Medicine, Stanford, CA 94305-5345. E-mail: madison@stanford.edu.

Dr. Pavlidis's present address: College of Physicians and Surgeons, Columbia Genome Center, Columbia University, 1150 St. Nicholas Avenue, New York, NY 10032.

Copyright (C) 2000 Society for Neuroscience $\quad 0270-6474 / 00 / 204497-09 \$ 15.00 / 0$ of LTP: injection of pharmacologically active substances into neurons. For presynaptic cells, this requires simultaneous recording from single synaptically coupled presynaptic and postsynaptic cells (Miles and Poncer, 1996). Previously (Pavlidis and Madison, 1999), we characterized synaptic transmission in such recordings between CA3 pyramidal neurons in organotypic hippocampal slices (see also Debanne et al., 1996). The most important findings from this previous work, in the context of LTP and the goals of this paper were as follows: (1) paired-pulse depression was much more frequently observed than facilitation (see also Debanne et al., 1996); (2) raising bath calcium had little effect on the maximal EPSC obtained, despite causing an increase in the average EPSC; and (3) inclusion of BAPTA in the presynaptic whole-cell electrode caused a rapid block of transmission, with clear effects being observed within 20 min (Borst and Sakmann, 1996; Ohana and Sakmann, 1998). The first two findings suggest that the probability of release at these synapses is relatively high and places limits on the extent to which changes in the probability of release can increase transmission. The third finding demonstrates that the presynaptic injection experiments are feasible, at least for low-molecular weight substances. Here, we describe LTP in the synaptic connections between nearby pairs of CA3 pyramidal cells and examine the effects of presynaptic injection $\mathrm{H}-7$ and nicotinamide, which had been shown previously to block LTP when bath applied (Malinow et al., 1988; Schuman et al., 1994).

\section{MATERIALS AND METHODS}

Details of our pair recording technique have been published previously (Pavlidis and Madison, 1999). In brief, interface cultures of hippocampal slices were prepared as described previously (Stoppini et al., 1991). Tissue was prepared from 7- to 10-d-old Sprague Dawley rats. Cultures were used after 7-14 d in culture. Individual slice cultures were trans- 


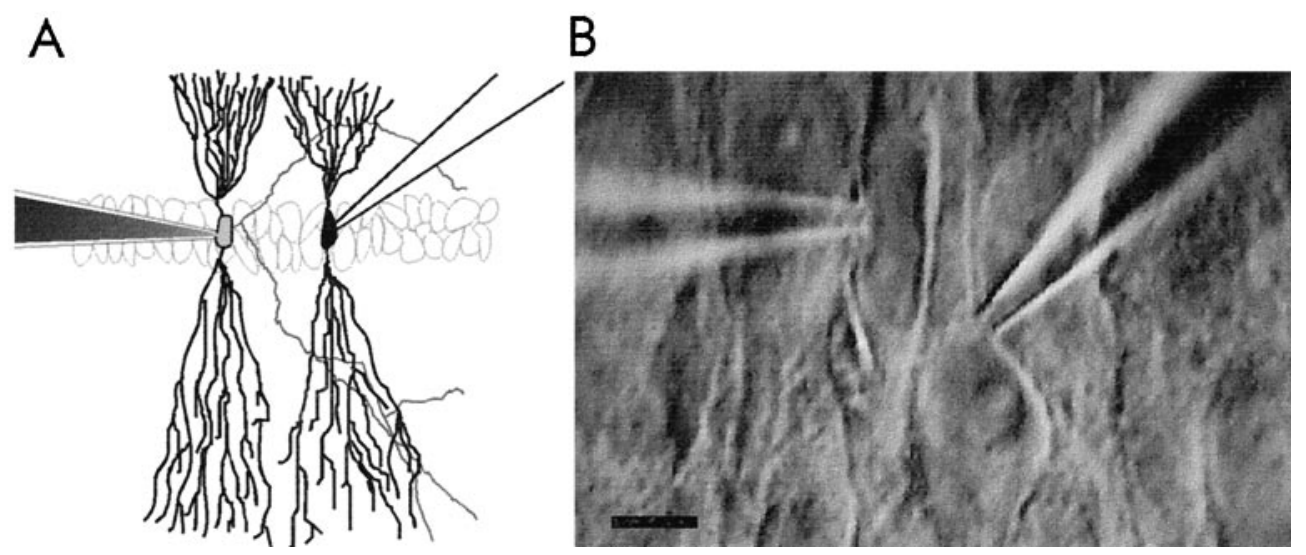

Figure 1. A, Schematic diagram of the recording configuration used in these experiments. Recordings from two neighboring pyramidal neurons were made in area CA3 of organotypic hippocampal slices. Postsynaptic cells were recorded using either the amphotericin perforated patch technique or standard whole-cell recording. The presynaptic cell was always recorded in standard whole-cell mode. In the later experiments in this study, pharmacologically active substances were introduced into the presynaptic cell by including them in the pipette internal solution. $B$, Recordings were obtained by observing the video image of the CA3 pyramidal cell layer in organotypic slices using differential interference contrast optics with infrared illumination. Electrodes were placed using a highly accurate micromanipulator (MP-285; Sutter Instruments, Novato, CA) and pressed against the cell membrane under visual control. This panel shows such a video image with two electrodes already in place. Scale bar, $\sim 10 \mu \mathrm{m}$.

ferred to a recording chamber superfused at $2-3 \mathrm{ml} / \mathrm{min}$ with artificial CSF (ACSF) with the following composition (in mM): $\mathrm{NaCl} \mathrm{119,} \mathrm{KCl}$ 2.5, $\mathrm{CaCl}_{2} 2.5, \mathrm{MgSO}_{4} 1.3, \mathrm{NaH}_{2} \mathrm{PO}_{4} 1, \mathrm{NaHCO}_{3} 26.2$, and glucose 11, $\mathrm{pH} 7.4$, saturated with $95 \% \mathrm{O}_{2}-5 \% \mathrm{CO}_{2}$ at room temperature $\left(21-23^{\circ} \mathrm{C}\right)$. ACSF reagents were of molecular biology grade (Fluka, Milwaukee, WI), which appeared to be important for obtaining reliable LTP.

Whole-cell recordings from CA3 pyramidal cells were made blindly (Blanton et al., 1989) or with the electrode being placed under visual control using an infrared-differential interference contrast microscope (Dodt and Zieglgansberger, 1990). Presynaptic and postsynaptic events were sampled at $10 \mathrm{kHz}$ and low-pass filtered at $1-2 \mathrm{kHz}$. Series and input resistance of voltage-clamp recordings were monitored throughout experiments and did not vary by $>20 \%$ over the course of the recording within experiments included in the data set. Perforated patch recordings were performed using amphotericin (Fluka) at $200-300 \mu \mathrm{g} / \mathrm{ml}$ (from a 60 $\mathrm{mg} / \mathrm{ml}$ DMSO stock) in a modified pipette solution: (in $\mathrm{mm}$ ) Cs methansulfonate 55, $\mathrm{Cs}_{2} \mathrm{SO}_{4} 75$, HEPES 10 , and $\mathrm{MgCl}_{2} 8$, pH 7.2 with $\mathrm{CsOH}$. The same solution without amphotericin was used to fill the tips of electrodes (2-5 M $\Omega$ ), whereas the amphotericin solution was used for backfilling. Amphotericin reached the tip of the pipette by diffusion within minutes. After formation of a $G \Omega$ seal between electrode and pipette, series resistance decreased as amphotericin inserted into the membrane, stabilizing within $10-60 \mathrm{~min}$ at between 15 and $40 \mathrm{M} \Omega$. In experiments using broken-patch whole-cell mode, series resistance was between 10 and $25 \mathrm{M} \Omega$.

To establish a pair recording, a second whole-cell recording was obtained in an adjacent area of the CA3 cell body layer (typically $\sim 100-300 \mu \mathrm{m}$ separation between cells in blind recordings and 10-100 $\mu \mathrm{m}$ in visualized recordings). The presynaptic electrode solution composition was (in mM): $\mathrm{K}$ gluconate 120 , HEPES $40, \mathrm{MgCl}_{2}$ 5, NaATP 2, and NaGTP 0.3 , pH 7.2 with KOH. Presynaptic cells were held in current clamp and induced to fire single action potentials by brief injection of depolarizing current (typically $20-50 \mathrm{pA}$ for $20 \mathrm{msec}$ ). When a successful pair was obtained (i.e., a monosynaptic EPSC was evoked by a presynaptic action potential), the presynaptic cell was stimulated by current injection at $0.03-0.1 \mathrm{~Hz}$ throughout the experiment. A connection was judged to be monosynaptic when the synaptic delay was $\leq 3 \mathrm{msec}$ after the presynaptic action potential and remained at a consistent latency from trial to trial (Pavlidis and Madison, 1999). Failures were trials indistinguishable from prestimulus baseline and were confirmed by slicing histograms (Pavlidis and Madison, 1999). Because the exact time of action potential occurrence during the depolarization of the presynaptic cell could vary slightly from trial to trial, analysis windows used for the postsynaptic EPSC were locked to the time of occurrence of the peak of the action potential. Sweeps in which no presynaptic action potential occurred or in which the postsynaptic recording was distorted by spontaneous synaptic activity were excluded from analysis. In some experiments, polysynaptic events obscured the peak of the event in many sweeps, so in these cases, the initial slope of the event was analyzed rather than the amplitude. LTP was induced by pairing presynaptic action potentials, evoked by presynaptic current injection, at $1 \mathrm{~Hz}$ for 1 min with postsynaptic depolarization to $-10-0 \mathrm{mV}$. Unless otherwise stated, the level of LTP was measured at $40 \mathrm{~min}$ after pairing.

To test the effects of presynaptic electrode contents on LTP, two different approaches were used. In the first, recordings were performed as above, obtaining a postsynaptic cell first in perforated patch mode, followed by a presynaptic cell in standard whole-cell mode. In this manner, we were able to follow the effects (if any) of presynaptic dialysis on baseline transmission without washing out the ability to induce LTP from the postsynaptic cell. However, because of the technical difficulty of maintaining recordings for long enough to obtain sufficient baseline and sufficient post-LTP records, we adopted a simplified technique for some later experiments. In these experiments, the presynaptic cell was obtained first, $30 \mathrm{~min}$ were allowed to elapse to permit diff usion of substances into the presynaptic cell, and then putative postsynaptic cells were acquired in whole-cell mode until one was found that was coupled to the presynaptic cell. Usually, this resulted in a successful pair recording within $10 \mathrm{~min}$. However, this was variable, leading to varying times of presynaptic dialysis. We adopted a standard of a minimum of $35 \mathrm{~min}$ of presynaptic dialysis before testing for LTP. Note that a $30 \mathrm{~min}$ period of dialysis with BAPTA in the presynaptic pipette was invariably effective in blocking transmission, demonstrating that this is sufficient time for small molecules to diffuse to the presynaptic terminal (Pavlidis and Madison, 1999). A brief baseline was then collected (5 min), and then the LTP induction protocol was applied. If a connected pair recording was not established within $30 \mathrm{~min}$, the presynaptic recording was terminated; thus, presynaptic dialysis times were $35-65 \mathrm{~min}$ at the time of LTP induction (35-70 min in control).

Stock solutions of H-7 (Calbiochem, La Jolla, CA) and nicotinamide (Sigma, St. Louis, MO) were prepared daily in water and diluted into the pipette internal solution.

\section{RESULTS}

\section{LTP at CA3 associational synapses in organotypic slice cultures}

Because our objective was to influence the synaptic terminals of presynaptic cells by injection of substances into the soma, we sought to study the effects of presynaptic manipulations in cells with a short axonal connection to their postsynaptic partner to minimize the distance substances had to diffuse along the axon. Thus, we performed these experiments at the synapses that CA3 pyramidal cell form with each other. This allowed us to record from neighboring cells, maximizing the chances that the connect- 
ing axon would be relatively short (Fig. 1). We used organotypic cultures because they exhibit more interconnectivity than acute slices, making it easier to obtain pairs of connected cells.

Because LTP at these CA3-CA3 synapses has not been well characterized, particularly in this type of cultured slice, we first studied the basic properties of this potentiation (see also Debanne et al., 1998, 1999). Our initial experiments were to test whether LTP could be reliably induced in area CA3 of organotypic slices. Whole-cell recordings were made from CA3 pyramidal cells, and synaptic currents were evoked with a bipolar stimulating electrode placed in stratum radiatum, near the CA3-CA1 border. The currents were complex, with monosynaptic and polysynaptic excitatory and polysynaptic inhibitory components, so the initial slope of the current was used as a measure of synaptic strength. We found that pairing postsynaptic depolarization to $-10 \mathrm{mV}$ while stimulating presynaptic fibers at $1 \mathrm{~Hz}$ for 1 min reliably induced LTP of $172 \pm 19 \%$ (mean + SEM, measured 40 min after pairing) (Fig. $2 A$ ). LTP was obtained in 12 of 15 attempts. The potentiation usually had a phasic time course, reaching an early peak averaging $200 \pm 15 \%$ within the first half hour after pairing before decaying to a stable level. This potentiation was blocked by $50 \mu \mathrm{M}$ D,L-APV, a selective NMDA receptor antagonist ( $n=2$; data not shown).

\section{Properties of LTP in pairs}

We then examined LTP induced between individual pairs of synaptically coupled CA3 pyramidal cells. For most of these experiments, the recording from the presumptive postsynaptic cell was obtained first, using the amphotericin perforated patch technique. Whole-cell recordings were then obtained from a series of putative presynaptic cells until one was found that was monosynaptically coupled to the previously acquired postsynaptic cell. Monosynaptic EPSCs could be evoked in $\sim 30 \%$ of tested pairs when the putative presynaptic cell was induced to fire an action potential by current injection. EPSCs were judged to be monosynaptic when it occurred in the postsynaptic cell at a short ( $\leq 3 \mathrm{msec}$ ), constant latency relative to the presynaptic action potential. Pairs displaying only polysynaptic potentials were discarded.

When presynaptic action potentials evoked at $1 \mathrm{~Hz}$ were paired with postsynaptic depolarization (by current injection), LTP of the unitary EPSC was induced ( $>20 \%$ potentiation in 24 of 32 experiments). This LTP usually developed over a period of several minutes after pairing was completed. Like the LTP of extracellular-evoked EPSCs, LTP between individual neurons often had an "early peak" that was followed by a decline to a stable, potentiated level. Overall, an average of $227 \pm 38 \%$ LTP was observed, measured at $40 \mathrm{~min}$ after induction. The early peak level of potentiation averaged $268 \pm 33 \%$. This LTP lasted for the duration of the recording (over $2 \mathrm{hr}$ in some cases).

We found that the LTP in these pairs is identical to the NMDA receptor-dependent LTP observed at CA3-CA1 synapses in acutely prepared slices. This conclusion comes from several lines of evidence. First, we noted that LTP was not induced if the postsynaptic cell was held in a standard whole-cell configuration for longer than $\sim 10 \mathrm{~min}$; that is, dialysis of the postsynaptic cell causes "washout" of some cytoplasmic factor required for LTP, as is found in acute slices (Malinow and Tsien, 1990) (data not shown). Second, LTP was blocked by bath application of APV $[n=5$; after pairing, EPSCs averaged $111 \pm 5 \%($ mean $+\mathrm{SE})$ of control] (Figure $3 A$ ). After removal of APV, LTP could be induced (APV washout tested on three pairs; subsequent LTP
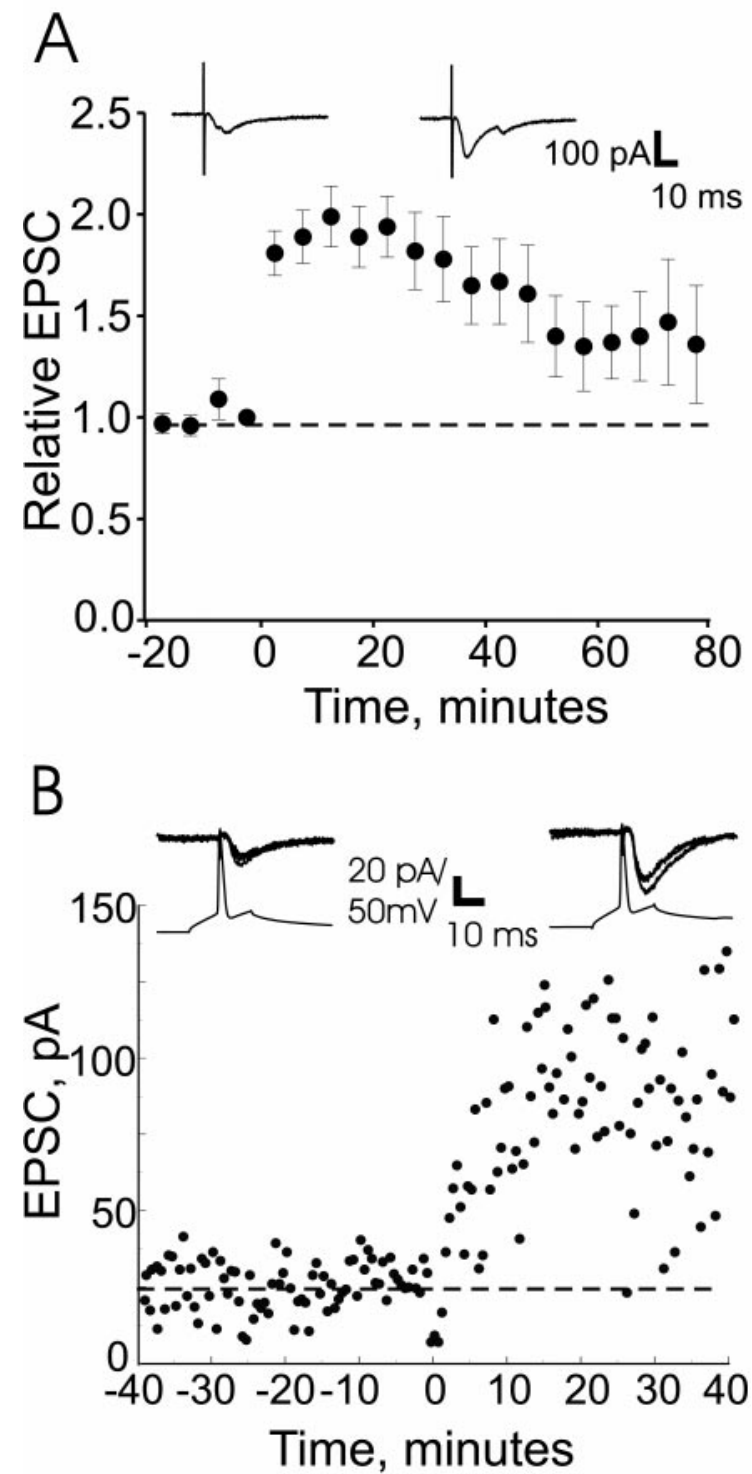

Figure 2. A, Associational synapse LTP in organotypic slices. EPSCs were recorded from CA3 pyramidal cells in whole-cell perforated patch mode while stimulating associational fibers with a bipolar stimulating electrode. At time 0, a 1 min pairing protocol (presynaptic action potentials at $1 \mathrm{~Hz}$ paired with postsynaptic depolarization to $-10-0 \mathrm{mV}$ ) was delivered. The average of 15 experiments is shown. The traces are from an individual experiment before (left) and after (right) LTP induction. B, CA3-CA3 LTP in pair recordings. An example of LTP in a pair is shown. At time 0 , postsynaptic depolarization to $-10 \mathrm{mV}$ was paired with $1 \mathrm{~Hz}$ presynaptic stimulation for $1 \mathrm{~min}$. The inset sweeps show traces of presynaptic action potentials and monosynaptic EPSCs before (left) and after (right) pairing.

was $175 \pm 41 \%$ ) (Fig. $3 A$ ). Third, LTP depended on simultaneous postsynaptic depolarization and presynaptic stimulation. Delivering either depolarization or stimulation at $1 \mathrm{~Hz}$ for $1 \mathrm{~min}$ alone resulted in no potentiation $(n=3)$ (Fig. $3 B)$. Thus, LTP is associative, as expected from its NMDA receptor dependence. Fourth, the LTP was pathway-specific. To test this, we used an extracellular bipolar electrode (as in Fig. 2) to stimulate a second independent pathway. The extracellular stimulus was set such that the presynaptic cell was not directly stimulated antidromically, ensuring that the pathways were entirely independent. LTP in either pathway did not have any effect on the size of the EPSC 

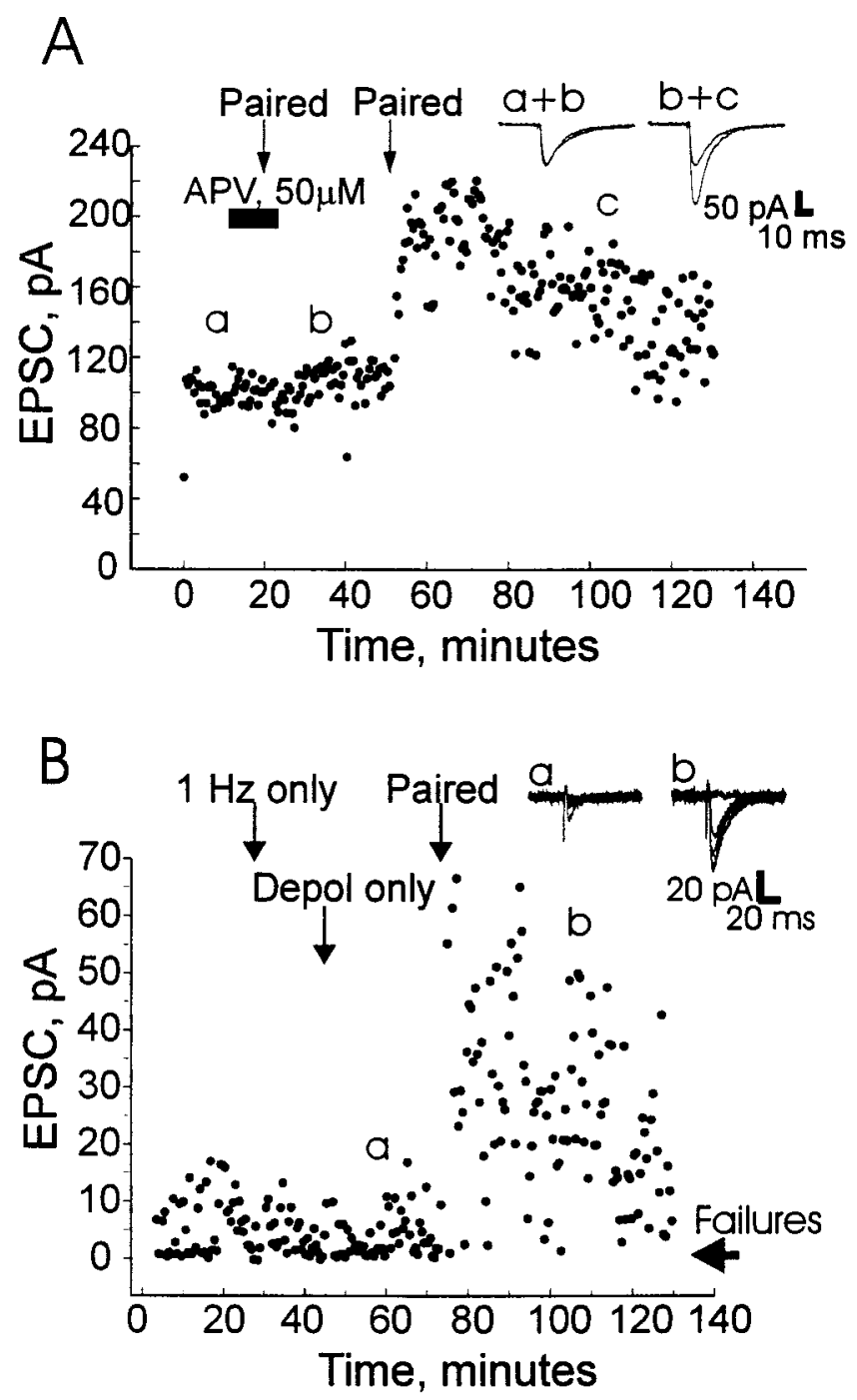

Figure 3. A, NMDA receptor dependence of LTP in a pair recording. Application of APV $(50 \mu \mathrm{M})$ completely blocked pairing-induced LTP. After washout of the APV, LTP was successfully induced. Inset sweeps illustrate responses before and after pairing in APV and after washout. $B$, LTP in pairs is associative. Delivering $1 \mathrm{~Hz}$ stimulation for $1 \mathrm{~min}$ or postsynaptic depolarization had no lasting effect on responses in pair recordings. In this experiment, after delivery of $1 \mathrm{~Hz}$ alone and depolarization alone, LTP was induced when they were delivered together. This experiment also provides an example of the large magnitude of LTP that could be obtained. In addition, the frequency of failures was drastically decreased after successful LTP induction (points around 0 current). Inset sweeps show five overlaid responses from the times indicated.

in the other pathway ( $n=4)$ (Fig. 4). Thus, in all these properties (washout, NMDA receptor dependence, pairing dependence, and pathway independence), LTP in pairs was identical to NMDA receptor LTP observed in acute slices.

At Schaffer collateral synapses in acute slices, LTP results in an increase in the reliability of synaptic transmission, as assessed by analyzing failures of transmission before and after LTP induction (Malinow and Tsien, 1990). In pairs having a relatively small EPSC, failures were often evident. In 12 experiments in which the failure rate before LTP induction was appreciable ( $>25 \%)$, LTP was accompanied by a decrease in the rate of failures from an average of $45 \%$ to $17 \%$ (Fig. 5).

An important feature of this potentiation is that it is dramati-
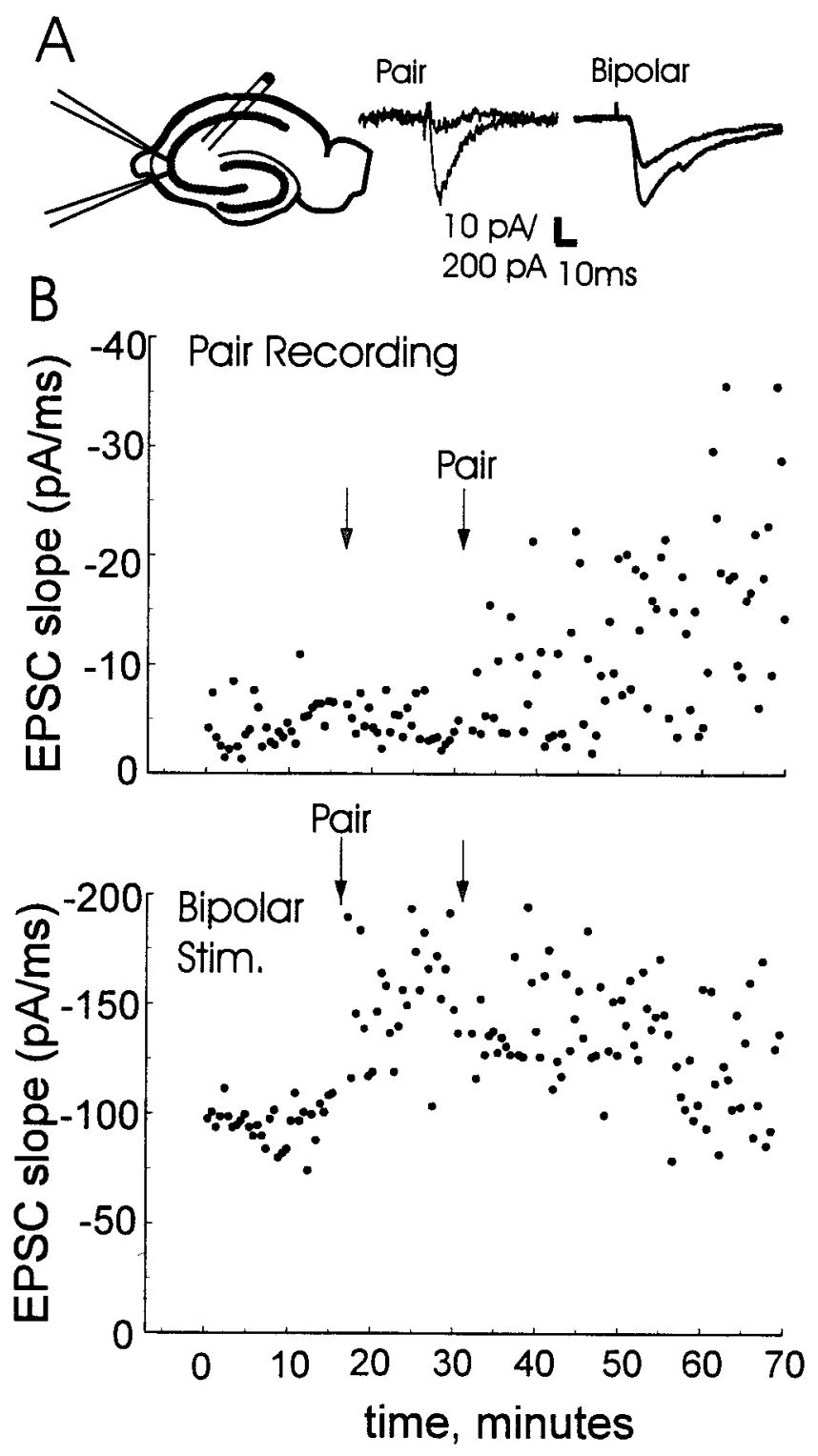

Figure 4. Pathway independence of LTP. $A$, The recording situation is illustrated schematically. A bipolar stimulating electrode in stratum radiatum was used to evoke responses from one pathway while recording from a pair. The traces are sample sweeps evoked from the pair (left) and using bipolar stimulation (right), before and after LTP induction in each pathway (overlaid). Calibration: $10 \mathrm{msec}, 10 \mathrm{pA}$ (Pair) and $200 \mathrm{pA}$ (Bipolar). B, Top, EPSCs evoked by stimulating the presynaptic cell. The gray arrow indicates when LTP was induced in the extracellularly stimulated pathway (shown in the bottom); there was no effect on the pair recording responses. At the black arrow, LTP was induced in the pair. In this experiment, slope was measured because of a contaminating polysynaptic inhibitory response. As shown in the bottom, this had no lasting effect on the extracellular-evoked EPSCs. This experiment is typical of five such experiments.

cally different than the increase in transmission obtained in these pairs by increasing the probability of transmitter release by raising extracellular bath calcium. We have shown previously that increasing the $\mathrm{Ca} / \mathrm{Mg}$ ratio from $\sim 2(2.5 \mathrm{~mm} \mathrm{Ca} / 1.3 \mathrm{~mm} \mathrm{Mg})$ to $\sim 7(5: 0.7)$ results in an increase in the average EPSC but very little change in the maximal EPSC amplitude (Pavlidis and Madison, 1999). In contrast to this "ceiling" effect, LTP in these pairs can result in large increases in the maximal EPSC obtained. This 


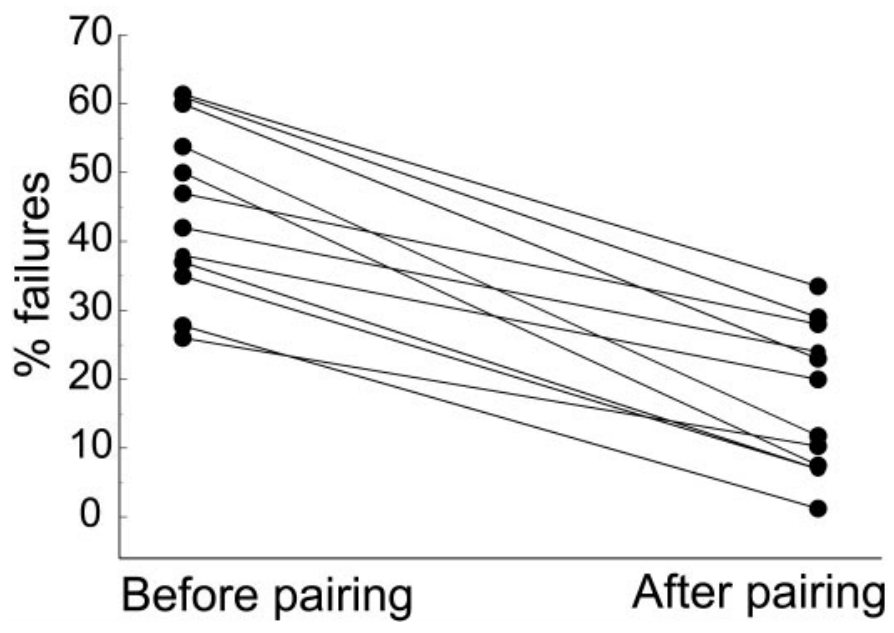

Figure 5. LTP reduces failures of transmission. In twelve experiments in which there was a high failure rate and in which LTP was successfully induced, a decrease in failure frequency was always observed. Each pair of points represents a single experiment before and after LTP.

phenomenon is illustrated in Figure 6, which shows examples of amplitude histograms before and after LTP.

\section{Presynaptic drug injection}

A main goal in these studies was to directly assess the role of the presynaptic terminal in producing LTP by injecting drugs into the presynaptic cell. We have demonstrated previously the feasibility of these experiments at this synapse by performing injections of calcium chelators (Pavlidis and Madison, 1999). For example, using $10 \mathrm{~mm}$ BAPTA, which blocks transmission, we found that clear effects on transmission could usually be observed within 10 min and within $30 \mathrm{~min}$ in all cases. Thus, for tests of presynaptic effects on LTP, we adopted a minimum 35 min waiting period after break-in of the presynaptic cell. We note that, over such time courses, presynaptic dialysis does not appear to affect synaptic transmission, and substantial LTP is still obtained (Fig. 2B). As an additional test of this, we determined the amount of LTP obtained in the three longest control experiments in which the presynaptic cell was held for 55-70 min before LTP induction. The LTP obtained in these three experiments $(209.4 \pm 24 \%)$ was not significantly different from the control average. Thus, our control electrode solution, which contains ATP and GTP, is compatible with synaptic transmission and LTP.

We have tested two compounds for effect on LTP. First, we used nicotinamide, an inhibitor of ADP-ribosyltransferase (Rankin et al., 1989). In a previous study, nicotinamide was found to block LTP when bath applied but not when injected into the postsynaptic cell, suggesting that its action may be presynaptic (Schuman et al., 1994). To test this directly, we included $30 \mathrm{~mm}$ nicotinamide in the presynaptic recording electrode $\left[\mathrm{IC}_{50}\right.$ for mono-ADP-ribosyltransferase is $3.4 \mathrm{~mm}$ and for poly-ADPribosyltransferase is $31 \mu \mathrm{M}$ (Rankin et al., 1989)]. We first observed that nicotinamide was associated with a significant decline in baseline transmission of $\sim 60 \%$ in three of five experiments (Fig. 7). Despite this, LTP appeared normal, with levels of potentiation comparable with control runs without nicotinamide $(227 \pm 57$ vs $246 \pm 37 \%$ in controls, measured $35 \mathrm{~min}$ after induction), and LTP of at least $120 \%$ was obtained in four of five attempts. The lone failure to obtain LTP was in one of the pairs in which nicotinamide appeared to decrease transmission. On the
A
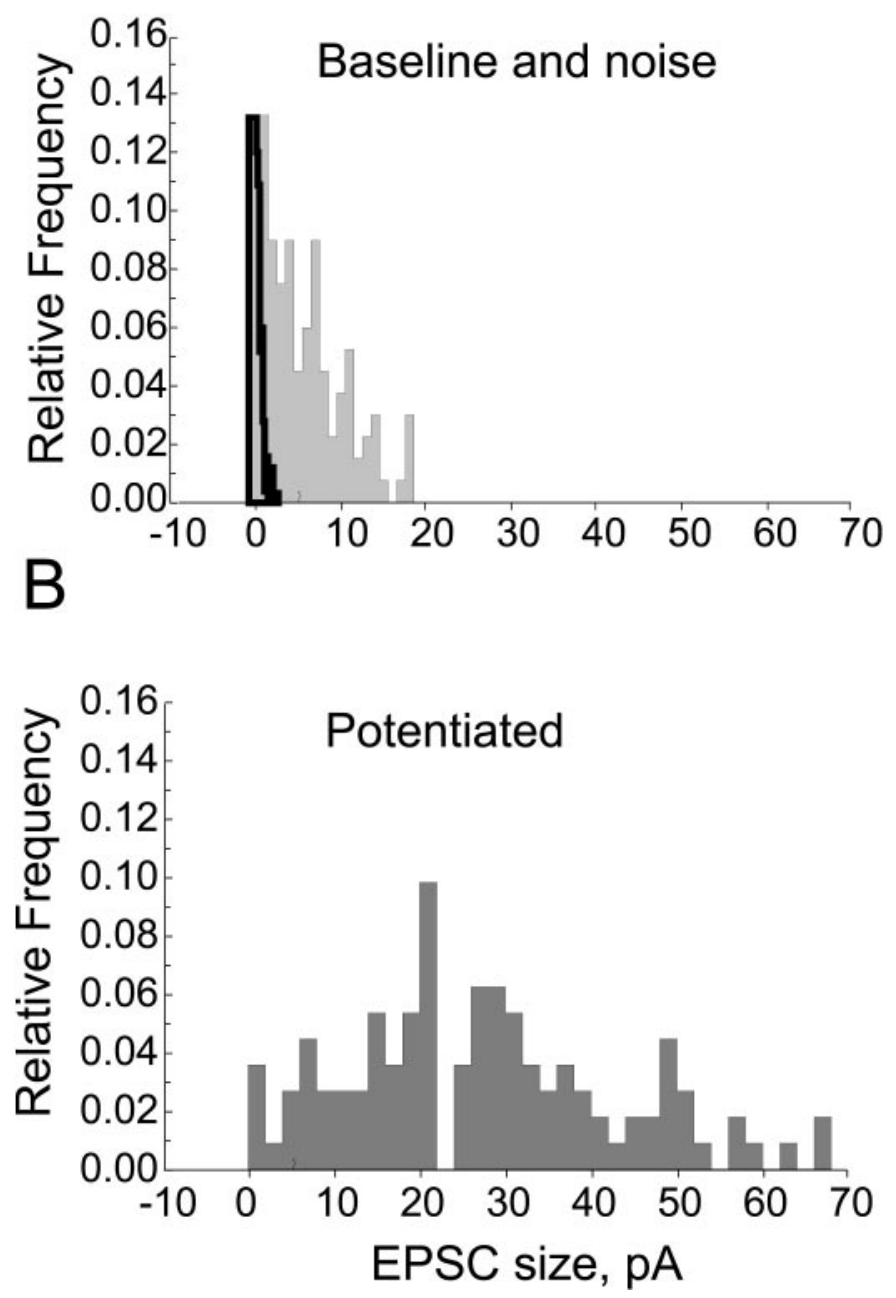

Figure 6. LTP results in large increases in potency. $A$, Histograms representing EPSC amplitudes and superimposed baseline noise for comparison in a single LTP experiment. $B$, EPSC amplitude distribution after inducing LTP in the above cell. This is typical of results obtained in pair recordings.

other hand, LTP of $>300 \%$ was obtained in the other two experiments in which transmission was inhibited by nicotinamide. Thus, even in those experiments in which there was a detectable presynaptic effect of nicotinamide on baseline transmission, showing that nicotinamide had successfully entered the cell, there was no indication that LTP was reduced.

We then tested the effect of $\mathrm{H}-7$, a nonspecific serine-threonine protein kinase inhibitor, by putting it in the presynaptic electrode at $100 \mu \mathrm{M}[\mathrm{H}-7$ is effective at blocking PKC, PKG, and PKA with low $\mu \mathrm{M} \mathrm{Ki}$ (Hidaka et al., 1984)]. Unlike nicotinamide or BAPTA, H-7 had no discernable effect on transmission over an 80 min period $(n=10)$ (Fig. $8 A$ ). Likewise, presynaptic injection of $100 \mu \mathrm{M} \mathrm{H}-7$ had no effect on transmission during the $1 \mathrm{~Hz}$ stimulation that is used to induce LTP (Fig. 8B). However, presynaptic H-7 did significantly reduce LTP when compared with control experiments without presynaptic H-7 (LTP with H-7 was $122 \pm 13 \% ; n=23$; $p<0.05$; compared with control LTP of 

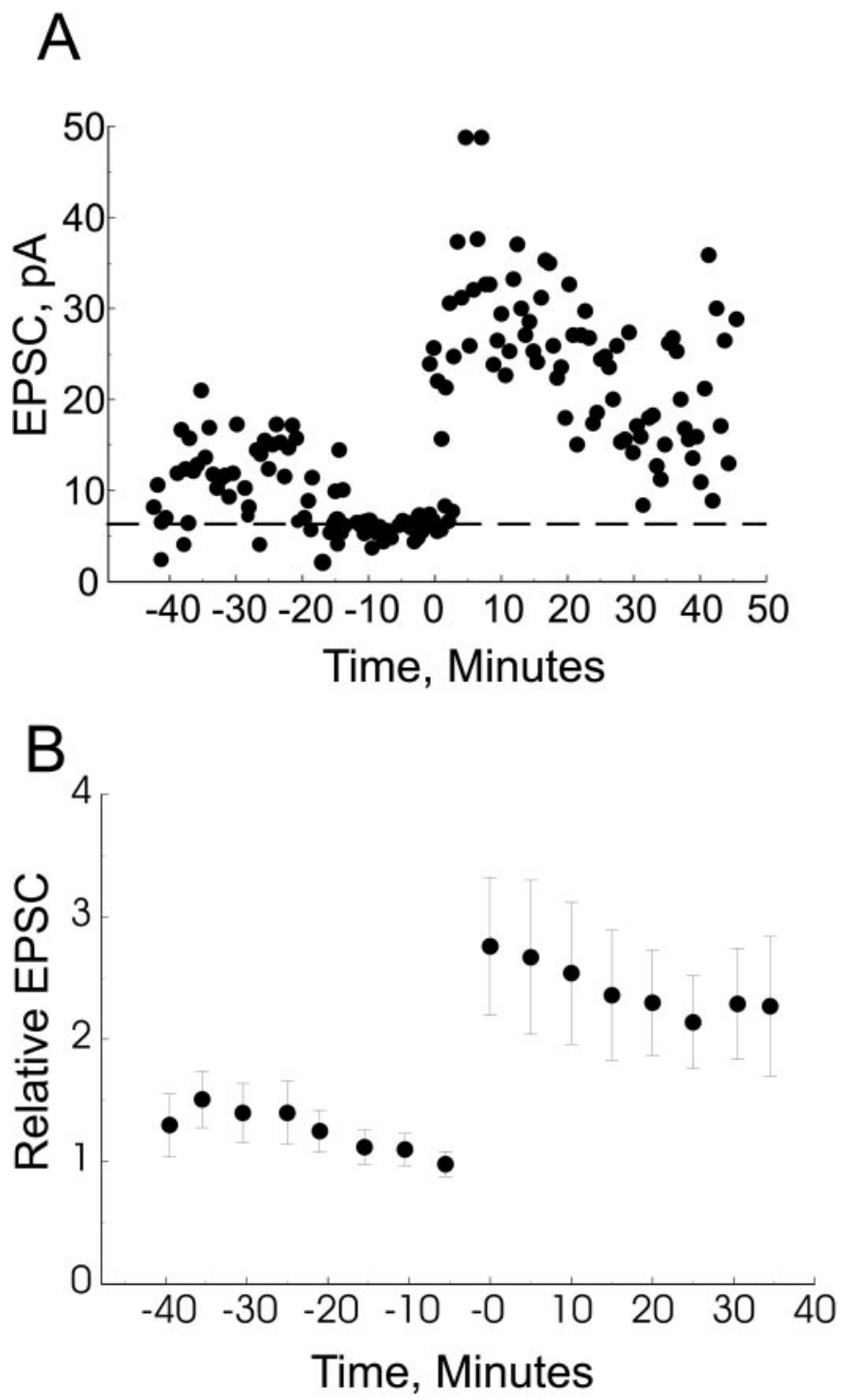

Figure 7. Presynaptic injection of an ADP-ribosylation inhibitor does not affect LTP. Nicotinamide was included in the presynaptic recording electrode at a concentration of $30 \mathrm{~mm}$. The depressive effect on basal transmission is clearly evident in both the example pair $(A)$ and in the average data $(B)$; however, LTP is essentially normal.

$227 \pm 38 \%$ measured 40 min after pairing) (Fig. $8 C, D$ ). The effect of $\mathrm{H}-7$ is apparent soon after pairing; early peak potentiation was $196 \pm 23 \%$ on average compared with controls $(268 \pm 33 \%$; $p<$ 0.05). In most experiments, the blockade of LTP was essentially complete, although in some experiments LTP was still obtained; LTP of $>120 \%$ of baseline was induced only 8 of 23 times $(35 \%)$ compared with 24 of 32 for controls (75\%).

As expected, postsynaptic injection of $\mathrm{H}-7$ also blocks the induction of LTP (106 $\pm 19.7 \%$ of baseline at $40 \mathrm{~min}$ after pairing; $n=5$ ) as has been shown previously (Malinow et al., 1989). We performed a control experiment to ensure that H-7 diffusion from the "presynaptic" electrode into the extracellular space, before obtaining a seal, did not cause a postsynaptic block of LTP. To mimic this extracellular application of $\mathrm{H}-7$, we placed an H-7-filled electrode $(100 \mu \mathrm{M})$ into the tissue between putative presynaptic and postsynaptic cells, until a whole-cell recording was obtained from a presynaptic cell with an electrode that did not contain H-7. Positive pressure was applied to this H-7-filled electrode to exactly mimic the $\mathrm{H}-7$ ejection that would normally occur from a presynaptic recording electrode. After successful acquisition of a synaptically connected pair, LTP was then induced. We found that LTP was normal in these pairs, averaging $211 \pm 15 \%$ (40 min after pairing; $n=5$ ). In this experiment, the additional $\mathrm{H}$-7-filled electrode was closer to the postsynaptic cell than usual, and H-7 was applied extracellularly using the same amount of positive electrode pressure but for 3-10 times longer than in presynaptic injection experiments. This rules out any potential extracellular and postsynaptic effects of $\mathrm{H}-7$ delivered via the presynaptic electrode during its transit to the cell.

\section{DISCUSSION}

In this paper, we have studied the properties of long-term potentiation of synaptic transmission between single pairs of CA3 pyramidal neurons. Long-term potentiation could be reliably induced in CA3-CA3 synapses in interface organotypic hippocampal slices, and this LTP appeared to be identical to the well known potentiation between CA3 and CA1 neurons. Long-term potentiation at these synapses is dependent on the NMDA receptor and has the properties of associativity and pathway independence expected for this type of LTP. Dialysis of the postsynaptic cell for a brief time before attempting LTP induction prevented the development of the potentiation. In addition, LTP at these synapses exhibited a decrease in the failure rate of synaptic transmission after the potentiation. Thus, it appears that LTP at CA3-CA3 synapses in these cultures is a valid model for the study of NMDA receptor-dependent potentiation.

There are many potential mechanisms for postsynaptic LTP expression, but presynaptic changes that support LTP must be expressed through the final common path of increased transmitter release. LTP in pairs was associated with a decrease in failure rate, which could be explained by an increase in the probability of release or by "awakening" of silent synapses (Isaac et al., 1995; Liao et al., 1995). Comparison of data presented in this paper and in Pavlidis and Madison (1999) suggests that increases in release probability cannot account for all of the increase in EPSC amplitude during LTP in these pairs. First, paired-pulse depression was prevalent in these synaptic pairs (see also Debanne et al., 1996; Pavlidis and Madison, 1999), suggesting that the probability of release is high for the majority of synapses. Furthermore, increasing release probability by raising extracellular calcium fails to increase the maximum amplitude of the EPSC recorded in a given pair (although it does increase the average size; Pavlidis and Madison, 1999). This amplitude ceiling is readily violated by LTP, which can increase the amplitude of the EPSC far beyond this ceiling. This suggests that LTP must reflect a factor, either presynaptic or postsynaptic, besides, or at least in addition to, an increase in the probability of release. We also cannot exclude a scenario in which all of the LTP is attributable to an increase in the probability of release, if probability was so low at some synapses that increasing calcium fails to reveal their presence in a limited sample of test responses. Such synapses would be effectively silent under normal conditions.

Investigations concerning the role of the postsynaptic cell have often used the approach of injecting substances into the postsynaptic cell to assay the effects of that manipulation on LTP (Lynch et al., 1983; Lledo et al., 1995). We have taken a similar approach to the study of potential presynaptic mechanisms, injection of substances directly into the presynaptic cell to assay their effects 

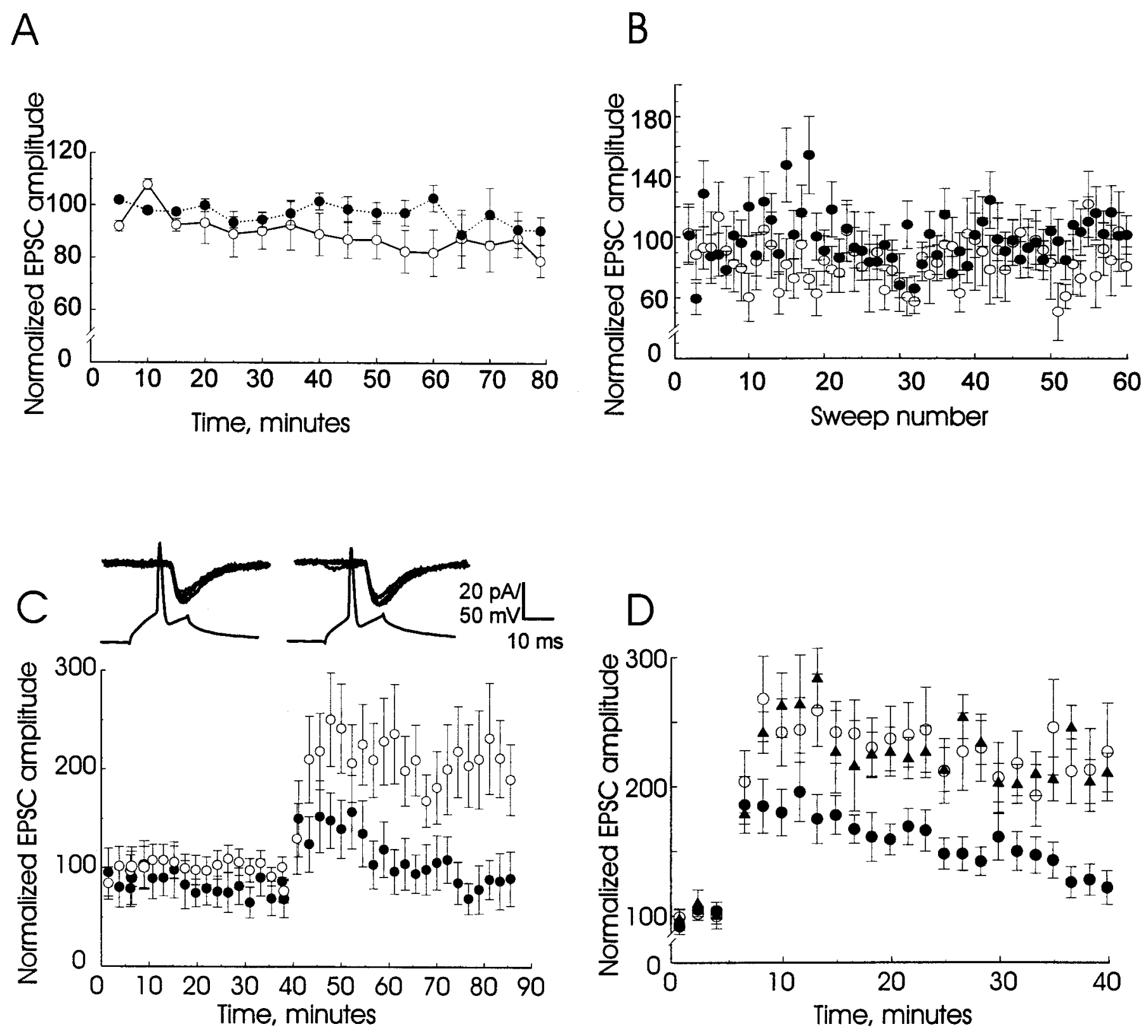

Figure 8. Presynaptic injection of the protein kinase inhibitor H-7 (100 $\mu \mathrm{M})$ inhibits LTP but does not affect basal transmission. Controls, open circles; H-7, filled circles. A, Monitoring basal transmission over a period of $80 \mathrm{~min}$. Injection of H-7 into the presynaptic neuron did not suppress basal EPSC amplitudes compared with control $(n=10)$. $B$, Responses to $1 \mathrm{~Hz}$ stimulation for $1 \mathrm{~min}$, such as those used to induced LTP, were unaffected by $\mathrm{H}-7$ injection into the presynaptic cell. $C$, Inhibitory effect of H-7 on LTP. The graph shows data from pairs in which the postsynaptic cell was obtained first with the amphotericin perforated patch technique, enabling basal transmission to be monitored for 40 min before pairing (see Materials and Methods). After pairing there was some initial potentiation, but this decayed rapidly leaving no significant potentiation after $20 \mathrm{~min}$. For $\mathrm{H}-7$ experiments, $n=7$; for control experiments, $10<n>17$. Inset, Sample sweeps from before and after pairing. $D$, Summary of all $\mathrm{H}-7$ experiments [both perforated patch and whole-cell mode (see Materials and Methods)] showing that LTP was reduced on average when compared with controls. The baseline data in this panel has been truncated to the length of the experiments with the shortest baselines.

on LTP. A similar technique has been used to investigate the mechanisms of synaptic potentiation induced by presynaptic cGMP injection in dissociated cultures (Arancio et al., 1995). However, it has not been applied previously to the study of LTP in slices, and there are no demonstrations of presynaptic pharmacological manipulations that block LTP in this system. Nicotinamide and $\mathrm{H}-7$ were selected for presynaptic injection because of their low molecular weight and relatively hydrophilic properties (which would help maintain a high intracellular concentration by limiting leakage out of the cell), and because both had been shown to block LTP when applied in the bath.

Nicotinamide, applied at a concentration of 1-10 $\mathrm{mm}$ in the extracellular medium, has been reported to completely prevent LTP via a blockade of a nitric oxide (NO)-activated ADPribosyltransferase. Based on the failure of postsynaptic nicotinamide injection to affect LTP, this activity did not appear to reside in the postsynaptic cell (Schuman et al., 1994). However, in the current experiments, presynaptic injection of nicotinamide 
completely failed to prevent or reduce LTP. Coupled with the previously reported lack of postsynaptic action (Schuman et al., 1994), one would have to conclude that the action of this agent is not in the presynaptic or postsynaptic pyramidal neuron. It is likely that nicotinamide reached the synaptic terminals based on both our experiences with BAPTA, showing the efficacy of putting substances into the terminal, and the observation that nicotinamide reduced transmission in most cases. Thus, our data are not supportive of a presynaptic role for ADP-ribosyltransferase in LTP. However, the fact that presynaptic nicotinamide did reduce basal transmission does lend support to the hypothesis that presynaptic NO-dependent processes participate in normal synaptic transmission (Meffert et al., 1994).

In contrast, inclusion of $\mathrm{H}-7$ in the presynaptic cell dramatically impaired LTP without suppressing basal transmission. This nonselective protein kinase inhibitor has been shown prevent the induction of LTP, but not to reverse it, when applied in either the bath or the postsynaptic cell (Malinow et al., 1988, 1989). Given the known postsynaptic effectiveness of $\mathrm{H}-7$ to block LTP, it is formally possible, but unlikely, that $\mathrm{H}-7$ may be leaking from the presynaptic terminal, crossing the synaptic cleft, penetrating the postsynaptic dendrite, and blocking LTP by inhibiting postsynaptic kinases. Blockade of LTP by bath application of $\mathrm{H}-7$ requires substantial bathing time at a high extracellular concentration (300 $\mu \mathrm{M}$ ), and the presynaptic terminal is a very small volume compared with the dilution volume of the extracellular space. Thus, the potential amount of $\mathrm{H}-7$ that could leak from a single presynaptic terminal is almost certainly too little to have a significant effect on the postsynaptic cell. Therefore, these H-7 results suggest a role for the presynaptic terminal in the production of LTP.

The most straightforward explanation for our results with $\mathrm{H}-7$ would be that this substance interferes with increases in presynaptic transmitter release associated with LTP expression. However, our own results comparing the effects of elevated extracellular calcium with LTP suggest that an increase in the probability of transmitter release cannot fully account for the expression of LTP. Thus, if LTP expression were presynaptic, it would have to involve additional mechanisms to increase transmitter output, such as increases in the number of vesicles, amount of transmitter per vesicle, or the kinetics of release from individual vesicles.

Other results in the literature have strongly suggested that the expression of LTP is at least in substantial part postsynaptic (Diamond et al., 1998; Luscher et al., 1998; Malenka and Nicoll, 1999). Can our results also be consistent with a postsynaptic expression of LTP? If the expression of LTP were found to be exclusively postsynaptic, this would necessarily limit the influence of presynaptic $\mathrm{H}-7$ to the induction or maintenance phases of LTP. Of these two, we believe it most likely $\mathrm{H}-7$ action is limited to the induction phase, because it is known that application of H-7 after LTP induction does not reverse established LTP (Malinow et al., 1988, 1989). Although the induction mechanisms of LTP are widely thought to reside within the postsynaptic cell, available evidence does not rule out participation of the presynaptic terminal in these processes. Previous reports (Malenka et al., 1988; Lledo et al., 1995; Yang et al., 1999) have suggested that postsynaptic experimental manipulations may be sufficient to induce LTP. For example, postsynaptic injection of $\mathrm{Ca}^{2+} /$ calmodulin-dependent protein kinase type II or of caged-calcium compounds (and subsequent photolytic calcium release) causes an increase in the strength of synaptic transmission that can occlude tetanus-induced LTP. One might argue that these experimental potentiations are not intrinsically persistent, but rather result from the continuous presence of the kinase or introduced calcium postsynaptically. This is clearly the case for injected kinase, and even with caged calcium, the time course of photolysis-elevated calcium can only be roughly estimated. But even if postsynaptic mechanisms are sufficient for inducing LTP, the presynaptic terminal can still participate in these processes in a permissive manner.

With current knowledge, it is impossible to know the precise nature of these putative presynaptic processes, but we can suggest some general possibilities. For example, the induction of LTP might require the release of a cotransmitter that is prevented by presynaptic H-7. Another possibility is that there is some kind of retrograde signaling between the postsynaptic and presynaptic cell that is necessary for induction. However, this model would require an additional step in which the presynaptic cell initiates subsequent anterograde signaling to the postsynaptic side, making this a model with more complex requirements. Third, cellcell interactions mediated by adhesion-type molecules between the presynaptic and postsynaptic cells may require regulation from both sides of the synapse to allow the induction of LTP (Tang et al., 1998). A requirement for this sort of a permissive coordination between the presynaptic and postsynaptic cell in the induction processes of LTP may explain the reported need for presynaptic activity to induce LTP (Zhuo et al., 1994) (but see Yang et al., 1999).

The data presented in this paper provide evidence that presynaptic protein kinases participate in the induction of LTP. Because $\mathrm{H}-7$ is a broadly effective protein kinase inhibitor, these experiments provide no information about the specific protein kinase(s) involved. Regardless of the exact action of H-7, these experiments provide the first evidence that an exclusive manipulation of the presynaptic cell can interrupt LTP. Thus, they comprise some of the most direct evidence to date for a role of the presynaptic terminal in this important process.

\section{REFERENCES}

Arancio O, Kandel ER, Hawkins RD (1995) Activity-dependent longterm enhancement of transmitter release by presynaptic $3^{\prime}, 5^{\prime}$-cyclic GMP in cultured hippocampal neurons. Nature 376:74-80.

Blanton MG, Lo Turco JJ, Kriegstein AR (1989) Whole cell recording from neurons in slices of reptilian and mammalian cerebral cortex. J Neurosci Methods 30:203-210.

Bliss TVP, Collingridge GL (1993) A synaptic model of memory: longterm potentiation in the hippocampus. Nature 361:31-39.

Borst JG, Sakmann B (1996) Calcium influx and transmitter release in a fast CNS synapse. Nature 383:431-434.

Debanne D, Guerineau NC, Gahwiler BH, Thompson SM (1996) Paired-pulse facilitation and depression at unitary synapses in rat hippocampus: quantal fluctuation affects subsequent release. J Physiol (Lond) 491:163-176.

Debanne D, Gahwiler BH, Thompson SM (1998) Long-term synaptic plasticity between pairs of individual CA3 pyramidal cells in rat hippocampal slice cultures. J Physiol (Lond) 507:237-247.

Debanne D, Gahwiler BH, Thompson SM (1999) Heterogeneity of synaptic plasticity at unitary CA3-CA1 and CA3-CA3 connections in rat hippocampal slice cultures. J Neurosci 19:10664-10671.

Diamond JS, Bergles DE, Jahr CE (1998) Glutamate release monitored with astrocyte transporter currents during LTP. Neuron 21:425-433.

Dodt HU, Zieglgansberger W (1990) Visualizing unstained neurons in living brain slices with infrared DIC-videomicroscopy. Brain Res 537:333-336.

Hidaka H, Inagaki M, Kawamoto S, Sasaki Y (1984) Isoquinolinesulfonamides, novel and potent inhibitors of cyclic nucleotide dependent protein kinase and protein kinase C. Biochemistry 23:5036-5041.

Isaac JTR, Nicoll RA, Malenka RC (1995) Evidence for silent synapses: implications for the expression of LTP. Neuron 15:427-434. 
Liao D, Hessler NA, Malinow R (1995) Activation of postsynaptically silent synapses during pairing-induced LTP in CA1 region of hippocampal slice. Nature 375:400-402.

Lledo PM, Hjelmstad GO, Mukherji S, Soderling TR, Malenka RC, Nicoll RA (1995) Calcium/calmodulin-dependent kinase II and longterm potentiation enhance synaptic transmission by the same mechanism. Proc Natl Acad Sci USA 92:11175-11179.

Luscher C, Malenka RC, Nicoll RA (1998) Monitoring glutamate release during LTP with glial transporter currents. Neuron 21:435-441.

Lynch G, Larson J, Kelso S, Barrionuevo G, Schottler F (1983) Intracellular injections of EGTA block induction of hippocampal long-term potentiation. Nature 305:719-721.

Malenka RC, Nicoll RA (1999) Long-term potentiation: a decade of progress? Science 285:1870-1874.

Malenka RC, Kauer JA, Zucker RS, Nicoll RA (1988) Postsynaptic calcium is sufficient for potentiation of hippocampal synaptic transmission. Science 242:81-84.

Malinow R, Tsien RW (1990) Presynaptic enhancement shown by whole-cell recordings of long-term potentiation in hippocampal slices. Nature 346:177-180.

Malinow R, Madison DV, Tsien RW (1988) Persistent protein kinase activity underlying long-term potentiation. Nature 335:820-824.

Malinow R, Schulman H, Tsien RW (1989) Inhibition of postsynaptic PKC or CaMKII blocks induction but not expression of LTP. Science 245:862-866

Meffert MK, Premack BA, Schulman H (1994) Nitric oxide stimulates $\mathrm{Ca}^{2+}$-independent synaptic vesicle release. Neuron 12:1235-1244.
Miles R, Poncer JC (1996) Paired recordings from neurons. Curr Opin Neurobiol 6:387-394.

Ohana O, Sakmann B (1998) Transmitter release modulation in nerve terminals of rat neocortical pyramidal cells by intracellular calcium buffers. J Physiol (Lond) 15:135-148.

Pavlidis P, Madison DV (1999) Synaptic transmission in pair recordings from CA3 pyramidal cells in organotypic culture. J Neurophysiol 81:2787-2797.

Rankin PW, Jacobson EL, Benjamin RC, Moss J, Jacobson MK (1989) Quantitative studies of inhibitors of ADP-ribosylation in vitro and in vivo. J Physiol (Lond) 507:237-247.

Ryan TA, Reuter H, Smith SJ (1997) Optical detection of a quantal presynaptic membrane turnover. Nature 388:478-482.

Schuman EM, Meffert MK, Schulman H, Madison DV (1994) An ADPribosyltransferase as a potential target for nitric oxide action in hippocampal long-term potentiation. Proc Natl Acad Sci USA 91:11958-11962.

Stoppini L, Buchs PA, Muller D (1991) A simple method for organotypic cultures of nervous tissue. J Neurosci Methods 37:173-182.

Tang L, Hung CP, Schuman EM (1998) A role for the cadherin family of cell adhesion molecules in hippocampal long-term potentiation. Neuron 20:1165-1175.

Yang SN, Tang YG, Zucker RS (1999) Selective induction of LTP and LTD by postsynaptic $\left[\mathrm{Ca}^{2+}\right]_{\mathrm{i}}$ elevation. J Neurophysiol 81:781-787.

Zhuo M, Hu Y, Schultz C, Kandel ER, Hawkins RD (1994) Role of guanylyl cyclase and cGMP-dependent protein kinase in long-term potentiation. Nature 368:635-639. 